\title{
An optical fiber loop sensor for vibration monitoring
}

\author{
A. W. Domański*, T. Poczęsny, K. Prokopczuk and P. Makowski \\ Faculty of Physics, Warsaw University of Technology, Koszykowa 75, 00-662 Warszawa
}

Received June 19, 2010; accepted June 24, 2010; published June 30, 2010

\begin{abstract}
In harmful environments such as refineries and factories, with a strong electromagnetic field, fire hazard and explosion risk there is a need to monitor the vibration of equipment and industrial infrastructure. Applying traditional measurement methods is a significant difficulty because of electric current which creates the risk of fire and influence of electromagnetic noise on these electronic devices. The solution to this problem is optical fiber measuring and monitoring methods. In this paper we present an optical sensor based on a fiber loop which can monitor vibrations and measure small relative displacements.
\end{abstract}

In environments such as refineries and factories there is a need to monitor the vibrations of equipment and industrial infrastructure. Additionally in such places there may be a risk of explosion or a fire hazard. Also in the vicinity of large drive machines there are strong electric and magnetic fields and electromagnetic noise [1-3]. Harmful effects of these fields on the operation of electronic devices are significant. Measurement methods which are based on electric effects create a fire hazard and are difficult to apply in places with a high explosion risk.

An optical fiber loop sensor can be used to measure relative displacement whereas signal analysis can be instrumental in measuring temporal velocity and total acceleration of the measured surface. Due to this fact it can monitor the vibration of machines and tested areas. The advantage of the presented sensor is a complete resistance to electromagnetic noise and both electric and magnetic fields in the measuring part as well as in the area of data transmission. It requires small optical power so that it does not create a fire hazard in the sensing part. An electronic analyzing system can be 500 meters away or more because the propagated light power does not leave the optical fiber. Signal transmission through optical lines does not suppress high-frequency components of a signal, which is a significant problem in the case of long electrical wires. Appropriate configuration of a fiber loop sensor allows monitoring low- and high-frequency vibrations and measuring a small displacement from sub millimeter scale up to several millimeters.

A fiber loop sensor works by using standard telecommunication optical fibers, single-mode or multi-

*E-mail: domanski@if.pw.edu.pl mode dependent on desired sensitivity. The advantage of this solution is the low cost of construction materials and a wide range of standardized connectors, light sources and detectors.

Fiber bends are one of the problems in telecommunication fiber optics. It leads to optical power losses in telecommunication lines and to disturbance of the signal. There are a few physical and mathematical theories modelling the power loss in bend fiber [4-6]. But all of them are based on the Marcuse bentloss formula (1) that was proposed in the 70's [4]:

$$
2 \alpha=\frac{\sqrt{\pi} \kappa^{2} \exp \left[-\frac{2}{3}\left(\gamma^{3} / \beta^{2}\right) R\right]}{2 \gamma^{3 / 2} V^{2} \sqrt{R}(\ln \gamma a)^{2}} .
$$

It describes the optical power loss $\alpha$ in bent fiber characterized by propagation and geometrical parameters ( $a$ - core diameter; $\beta, \gamma, \kappa, V$ - propagation constants and quantities describing waveguide) with the bending radius $\mathrm{R}$. The Marcuse bend formula was derived from the Coupled Mode Theory for optical waveguides.

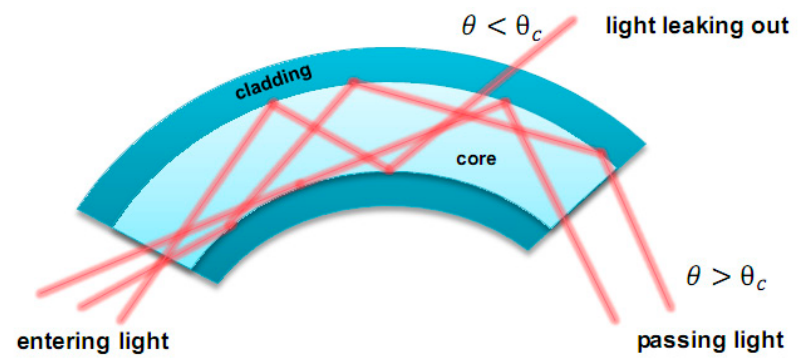

Fig. 1. Geometrical loss mechanism in bent fiber

In a geometrical way we can imagine that for some "light rays" propagating in the core the angle of incidence on the boundary of core and cladding is lower than the critical angle for total internal reflection. It happens because of the deformation of the fiber geometry caused by bending. These rays are leaking out and some part of the optical power is lost (Fig. 1).

Our idea is to take advantage of this effect by using the power losses as a useful signal and quantity corresponding to the bend radius. 
The simplest configuration of bent fiber is impractical, but by making a loop on the fiber we can change it into displacement sensing device. The loop touching the tested surface is deformed and it changes its bending radius. The operating principle of a fiber loop as a sensor is shown in Fig. 2.

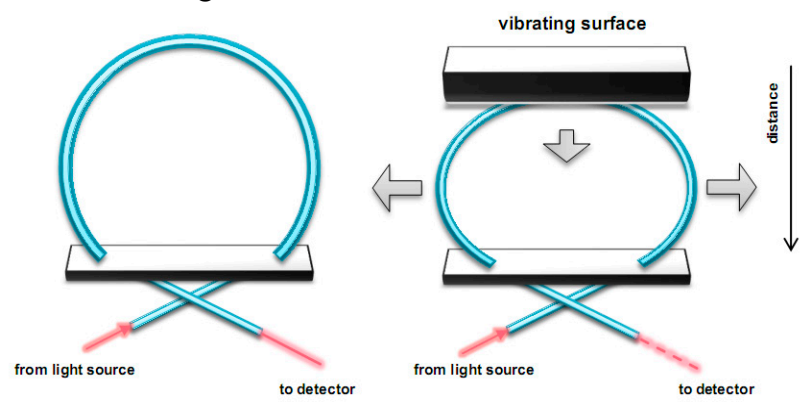

Fig. 2. Fiber loop as a vibration sensor - operating principle

By analyzing the output signal we can determine the position or the relative distance which the tested surface has moved. The response signal is very well characterized. It is constructed of three parts: near side, optical peak and far side. The near side has low sensitivity and can be treated as a "dead zone" region. The region of potential use characteristics is the far side which has a linear relationship to the compression of the loop (Fig. 3).

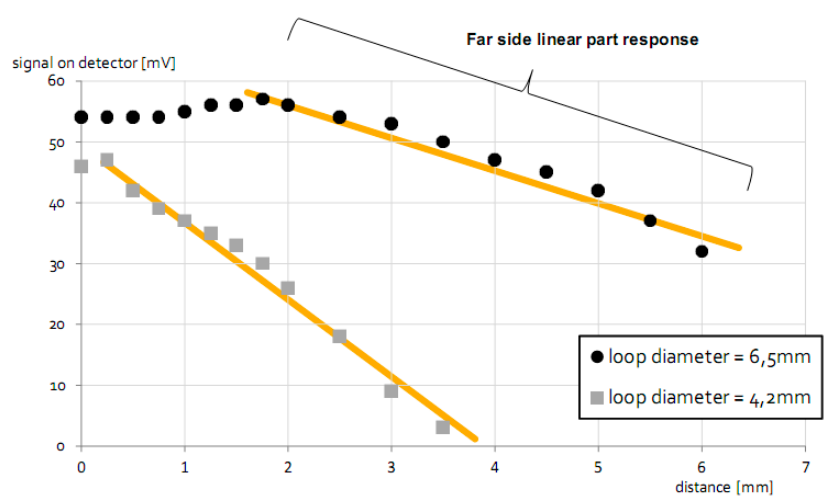

Fig. 3. Sensor characteristic

The shape of this response curve depends on loop diameter. The "dead zone" region is present only in fiber loops which have a large diameter. But even if we need large loop for high amplitude vibrations the "dead zone" region can be easily compensated by initial compression (position). The construction of a sensor head protects fiber against fracture and at the same it sets the maximum measurable displacement amplitude. If higher amplitudes occur the sensor will be "blinded" but the head and fiber will not be damaged.

The sensor can be calibrated by designating the response curve (Fig. 3) and defining the linear part on which the sensor works correctly. In this case, output voltage will be a linear function of surface displacement. By approximating this part of the response curve to linear function (where $U$ is the output voltage on a detector, $S$ is the sensitivity and $r$ is the displacement) it is possible to determine sensitivity $\mathrm{S}$, which is equal to:

$$
S=\frac{U}{r} \text {. }
$$

Finding the value of $S$ in a particular case is equivalent to the calibration of the sensor. From now on its output signal can be easily turned into a displacement.

The voltage level from the detector contains information about the surface position and its push on a fiber loop. After calibrating and determining the parameter $S$ fiber loop can be used as a vibration sensor giving information about the displacement, velocity and acceleration.

Standard piezoelectric vibration sensors measure the force which is proportional to acceleration. They find velocity and displacement by using integrating systems. The integration of low frequency signals is very difficult. The working principle in the case of a fiber loop is opposite - the sensor measures the light power loss proportional to the displacement and the system differentiates the signal to find velocity and acceleration:

$$
\begin{aligned}
& V(t)=\frac{d r}{d t}, \\
& a(t)=\frac{d^{2} r}{d t^{2}} .
\end{aligned}
$$

A significant advantage of the presented sensor is the wide range of measurable frequencies with a lack of troublesome resonance influence. It has been proved that fiber loop sensor is able to detect low and high frequency vibrations (Fig. 4).

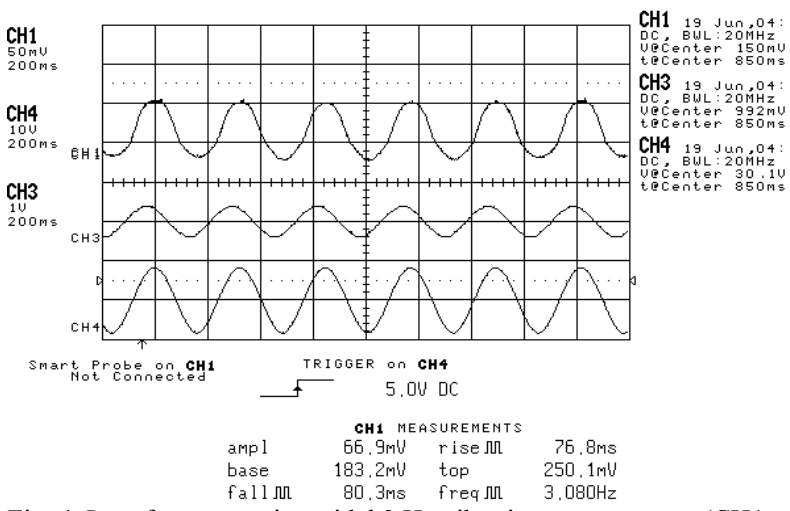

Fig. 4. Low frequency sinusoidal $3 \mathrm{~Hz}$ vibration measurement $(\mathrm{CH} 1-$ fiber loop sensor, $\mathrm{CH} 3$ - vibrating piezoelectric plate response, $\mathrm{CH} 4-$ signal from generator)

Measurement of low frequency signals is a major problem when piezoelectric sensors are used. With a fiber 
loop sensor there is no problem of measurable band limits. Also there is very good accuracy in sensing of vibrations with waveforms other than sinusoidal (Fig. 5, 6 ), which is sometimes hard to obtain with piezoelectric sensors.

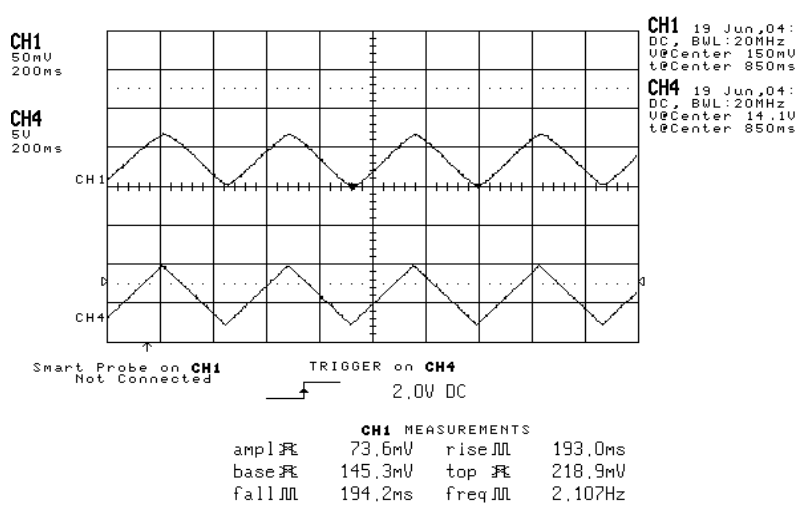

Fig. 5. Triangular $2 \mathrm{~Hz}$ vibration characteristic measured by loop sensor (CH1 - loop sensor, $\mathrm{CH} 4$ - signal from generator)

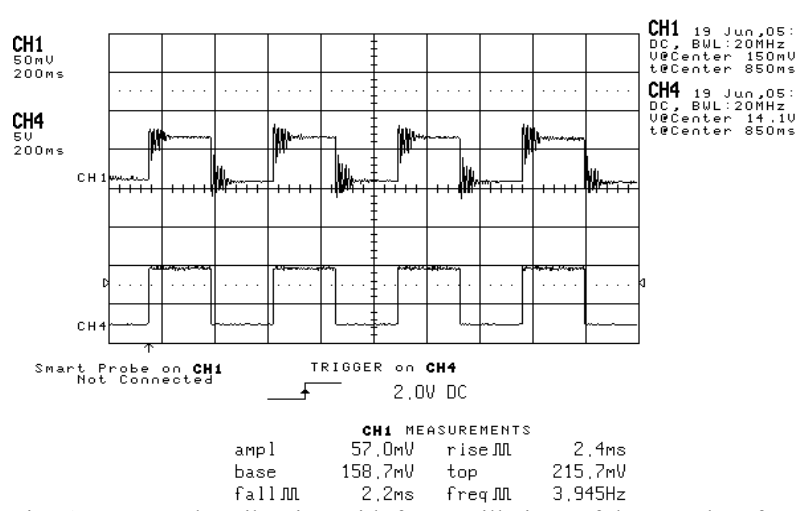

Fig. 6. Rectangular vibration with free oscillations of the tested surface (CH1 - loop sensor, $\mathrm{CH} 4$ - signal from generator)

The transmission spectrum of bent fiber is strongly dependent on any deflection. All propagation constants which are present in equation (1) are functions of the wavelength. Thus the shape of the transmission curve depends on the diameter of the loop and its deformations. Figure 7 and 8 present changes of spectrum for loop compression and characteristic periodical local maximums.

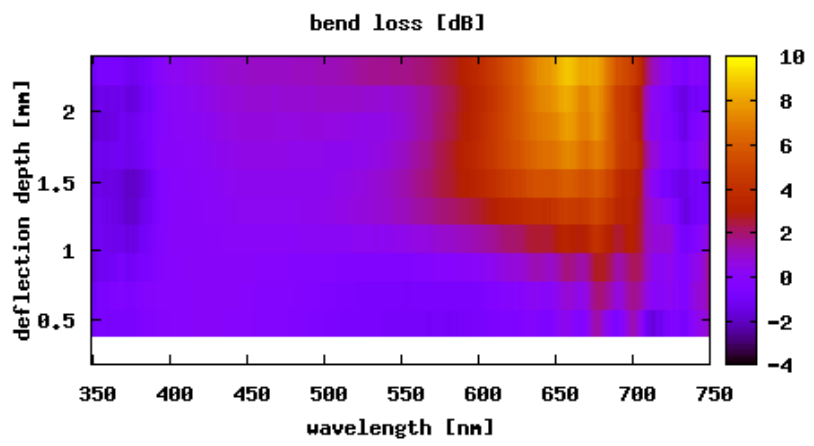

Fig. 7. Power losses in spectrum domain

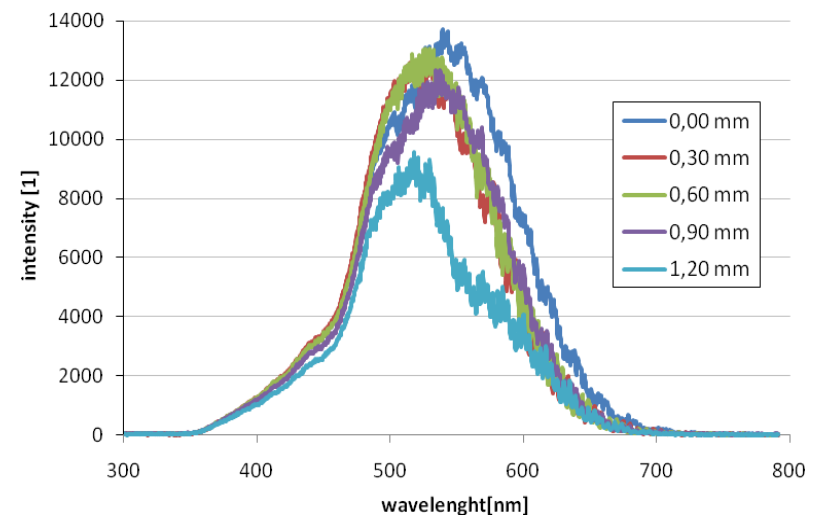

Fig. 8. Transmission spectra of deformed loop

It opens up the possibility to work with narrow bandwidth light sources on a small part of the fiber transmission spectrum (local maximums). This feature can be used to make cascade sensors. By making several loops and coupling light from different wavelength sources we can arrange multiple sensors on a single optical fiber.

This paper presents a new idea of vibration measurement system using a simple fiber loop. Furthermore we show the quality and accuracy of measurement in spite of its simplicity. This kind of sensor is able to measure very low vibration frequencies, which is significant in monitoring large machines and devices in factories and refineries.

This work was supported by the National Centre for Research and Development (NCBiR) under the grant $\mathrm{N}$ R01 0026 06/2009

\section{References}

[1] T.R. Woliński, D. Budaszewski, A.W. Domański, S. Ertman, G. Goleniewski, M. Wydmański, Proc. SPIE 6616, 66161U (2007).

[2] T.R. Woliński, A.W. Domański, D. Budaszewski, S. Ertman, Proc. SPIE 6585, 65850H (2007).

[3] G. Rajan,Y. Semenova, G. Farrell, Electr. Lett. 44, 1123 (2008).

[4] D. Marcuse, J. Opt. Soc. Am. B 66, 216 (1976).

[5] R.T. Schermer, J.H. Cole, IEEE J. Quantum Elect 43(10), Oct (2007).

[6] P. Wang, Q. Wang, G. Farrell, G. Rajan, T. Freir, J. Cassidy, Microwave and Opt. Techn. Lett. 49(9), 2133 (2007). 